\title{
Lgate-S: Simulation Media for Learning Logic Gate
}

\author{
Harsa Wara Prabawa ${ }^{1}$, Erni Nuraeni ${ }^{2}$, Rizky Rachman Judhie ${ }^{3}$, Jajang Kusnendar ${ }^{4}$ \\ \{harsawara@upi.edu ${ }^{1}$,erninr@student.upi.edu ${ }^{2}$, jkusnendar@upi.edu ${ }^{4}$ \} \\ Universitas Pendidikan Indonesia ${ }^{1,2,3,4}$
}

\begin{abstract}
This study tries to develop a learning type of simulation type because theoretically, simulation is considered more effective in helping students with high levels of anxiety in understanding a topic. That is because it presents a series of contextual objects/content that demands student involvement in conducting experiments. 84 Vocational High School students in the IT field in several schools in the city of Bandung tested this simulation media. From three times of media testing, the results obtained that the simulation media can make students better understand the concept of abstract logic material because simulations visualize the content on electric circuit illustrations that made as if the user is in a real situation. Nevertheless, the simulation media still need to be improved, especially in terms of the scenario of the presentation of the exercise and the complexity of the cases presented.
\end{abstract}

Keywords: Computer-assisted-instruction, Simulation, Logic gate

\section{Introduction}

Based on the 2013 curriculum, which applied in Vocational High Schools (SMK), particularly in the field of Information Engineering, Computer Systems is one of the compulsory subjects for SMK students majoring in Computer and Network Engineering (TKJ), Software Design (RPL), and Multimedia. This subject used as a basis for studying Computer Organization and Architecture, Network Operating Systems, Information Logic, and Digital Systems in the field of Informatics Engineering expertise. That way, the Computer System becomes very important because it is the foundation of Informatics Engineering materials. In general, there are two methods regarding the delivery of content in Computer System subjects, namely theory and practice. The theoretical study on Computer System material presented through classical learning. This is done because students need to understand the basic concepts of Computer Systems before; in the end, students practice them. The delivery of material that is practicum is done through the introduction and identification of hardware (hardware) directly by using the hardware components provided in the laboratory.

Most of the problems in the learning process of Computer Systems are usually caused by theoretical concepts that have not been correctly understood by students. This is partly thought to be caused by abstract ideas and learning activities that tend not to be contextual. The material presented tends to be theoretical and monotonous. The impact of the problem occurs when students proceed to the next level. Students will experience many difficulties when getting material related to Computer Systems because they have a not optimal understanding of previous Computer System learning.

Interviews conducted with several students at one of the Vocational Schools in Bandung revealed that many students who understood the practice-based material more than the theory- 
based material. One of the theory-based Computer System subject matter that is considered difficult by students is the Logic Gate material. The students felt the content was difficult to understand because they only paid attention to the texts, numbers, and symbols presented by the teacher on the media used. The material becomes abstract if it is not accompanied by a visualization of what presented. It makes students not understand and quickly bored in carrying out learning in class so that the impact on the acquisition of learning outcomes

The development of innovation in the field of information technology, no doubt, affects the development of the use of information technology in learning. One of them is by implementing Computer Assisted Instruction (CAI). CAI is computer-based learning that can make students interact and deal directly with computers individually so that what is experienced by a student will be different from what is experienced by other students [1] [2]. One type of CAI that often used is CAI simulation type, in addition to other types namely drill and practice, tutorials, games, and problem solving [3]

Simulation can understand as an imitation representation of the functionality of a system or process (http://www.merriam-webster.com/). Simulation can also be said as an experiment by doing a simple imitation using a computer on a particular order (and at a specific period time as well), to provide a better understanding of how the system works [4]

The concept of a simulation device (Visual Interactive Simulation - VIS) was first introduced by Hurrion (1976) as a computer model that presents a visual display in the form of animated moving objects [4]. The use of simulation tools in presenting information at least promises several benefits, including [4]: a) a better understanding of object patterns, this is because the device allows users to trace events that occur and identify potential deficiencies of the object; b) ease in verifying and validating the effectiveness of the system's operation; c) allows for an interactive process of experimentation, especially in the context of trying out new ideas to develop a problem or test a possibility; d) increased understanding of possible outcomes, this condition relates to the process of observing an event that can be carried out on an ongoing basis; e) allows it to use as a media for data recovery and communication aids in presenting information to a broader party; f) potentially used as a medium for problem-solving in groups. The simulation itself is an important part (of many other essential aspects) in learning computer science. This is because it can reach an understanding at the lowest level (only for the fulfillment of specific tasks) to the highest knowledge (e.g., system analysis) [5]

\section{Methods}

The research approach used in this study refers to the development research concept with an iterative type of software development framework. This approach is the development of the prototype model that is used in conditions when the software requirements developed will continue to improve. While the process of testing prototype simulation media uses a preexperimental scenario. This simulation media tested 84 Vocational High School students in the IT field in several schools in the city of Bandung.

\section{Results}

\subsection{Content analysis}


Logic Gate Material is a Computer System material that contains mathematical logic consisting of numbers, symbols, and tables. One of the points of learning objectives of the Logic Gate states that students are expected to be able to analyze the logic gate series, meaning students must be able to describe, differentiate, and sort the logic gate series to be classified according to specific criteria, then look for their links and interpret their meanings. To direct students in achieving learning objectives that refer to these essential competencies, material analysis carried out so that students can understand the material concepts easily. The following stages are the presentation of the content of the material that has analyzed:

1) First, the media used should be able to convey the primary element of the Logic Gate by using a brief theory and accompanied by an overview of the approach. The goal is that students can understand the main content of reading and make it easier for students to remember the material quickly.

2) Second, the media used should be able to explain the material of the Logic Gate with an illustration that can be simulated by students. The purpose of holding simulations and examples in a material presentation is so that students can connect abstract material into real situations, so students can find out the outputs contained in the truth table by pressing the inputs available on the media. With simulations, students can conduct experiments directly without the need for an actual environment, thus avoiding the risk of accidents.

3) Third, the media created should be able to explain the material of the Logic Gate with a picture of the logic gate symbol scheme whose input can be simulated by students. The goal is that students can know each logic gate symbol, know the input and output components of a symbol, and know the output results when students press available input.

4) Fourth, the media created should be able to explain the conclusion of the Logic Gate material. The goal is that students can know the relationship between the two simulations that have described, as well as knowing the core theory of each Logic Gate

\subsection{Technology analysis}

The technology used by teachers as teaching aids is only limited to PowerPoint media. The technology is considered less attractive by students because the material presented is only in the form of theories that explained textually, so learning in class is very monotonous and makes students feel bored. Also, the content of Logic Gate is material that is difficult to understand if the concept is only delivered theoretically because the idea of Logic Gate is abstract if it is not accompanied by visualization. Therefore, the researcher seeks to create new technology in the form of a simulation type CAI design, which is learning multimedia by using an imitation of a product that describes a real situation, so students will better understand the concept of teaching material. Simulations made involve illustrations and animations in them. The pictures and animations aim to illustrate a theoretical logic gate concept, so students are more interested in learning and can absorb material easily.

\subsection{Prototype design}

The primary material in this simulation is in the form of an explanation of the definition of logic gates, the definition of truth tables, and the classification of logic gates that need to be presented using a brief theory. Presentation of the content of this material intended as 
preliminary learning, so students can understand the main contents of reading before entering into a specific explanation, which consists of a description of each first logic gate and combination logic gate.

Material logic gates are not easy to understand if they are not accompanied by visualization. That way, it needs to add an image/animation to introduce how to describe a logic gate, truth table, along with the input and output of the truth table, which is usually only explained textually. The addition of images to a brief theory also intended so that students can remember the material quickly

The second design component is the logic gate content design. At the beginning of prototype design, the content of the Logic Gate material was designed to be an illustration of a tap water channel that the user could simulate. This simulation only intended for the next part of the content, which is an explanation of the types of logic gates and the conditions of input and output. If the illustration is related to Logic Gate material, then the data is illustrated with a tap connected to a channel, while the production illustrated with water coming out of a chain. Users can simulate the tap by opening or closing the valve. A closed tap is interpreted as input 0 , while an open tap interpreted as input 1 . A simulation conducted on the lid can produce an output in the form of the appearance of water. Water that does not appear is interpreted as output 0 , while water that seems to understand as output 1 .

From several prototype designs using water channels, researchers experienced a problem that is unable to describe the logic gate of XOR and XNOR using the illustration of a water tap channel. If the picture of the electrical circuit is related to the Logic Gate material, the input illustrated by a switch connected to the course, while a lamp shows the output, users can simulate the switch by pressing the switch found on the media. A switch with an OFF condition is interpreted as input 0 , while a switch with an $\mathrm{ON}$ condition interpreted as input 1. Simulation carried out on the switch can produce an output in the form of a lamp condition. If the lamp condition is off, it means that the output produced has a status of 0 . If the lamp condition is on, it means that the output produced has situation 1. Figure 1 illustrates the use of electronic circuits in describing the XOR concept.

Logic gate test results indicate an increase in learning outcomes in each class group consisting of three levels, namely the upper-class group, middle-class group, and lower-class group. From these results, it has known the increase in value (gain) of the upper-class group by 0.47 , the middle-class group by 0.58 , and the lower class group by 0.58 . Each group included an increase in the medium category.

The overall average gain results also stated included in the medium category, which is equal to 0.56 , meaning that the use of CAI in the Logic Gate learning process has a positive influence on student learning outcomes.

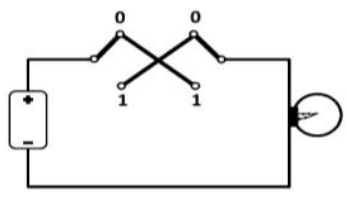

(a)

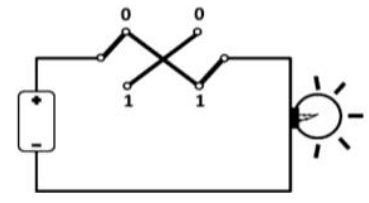

(b) 


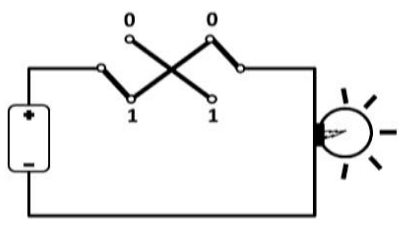

(c)

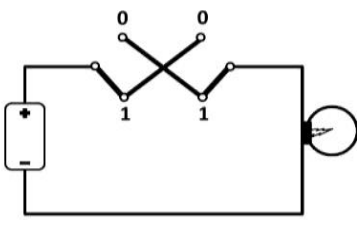

(d)

Fig. 1. Illustration of XOR Logic Gate presentation.

Figure 2 displays the media interface about the illustration of the electrical circuit, the truth table, and an explanation of the simulation performed. Students can do the simulation by pressing the switches on the page. If the switch pressed, the lamp conditions, truth table, and explanation would change according to the switch conditions. In the simulation, there is an explanation button that will open as a pop-up if students have pressed the button. The simulation explanation page 1 displays an explanation of how the electrical circuit worked when the switch pressed. The explanation pop-up display can be seen in Figure 3.

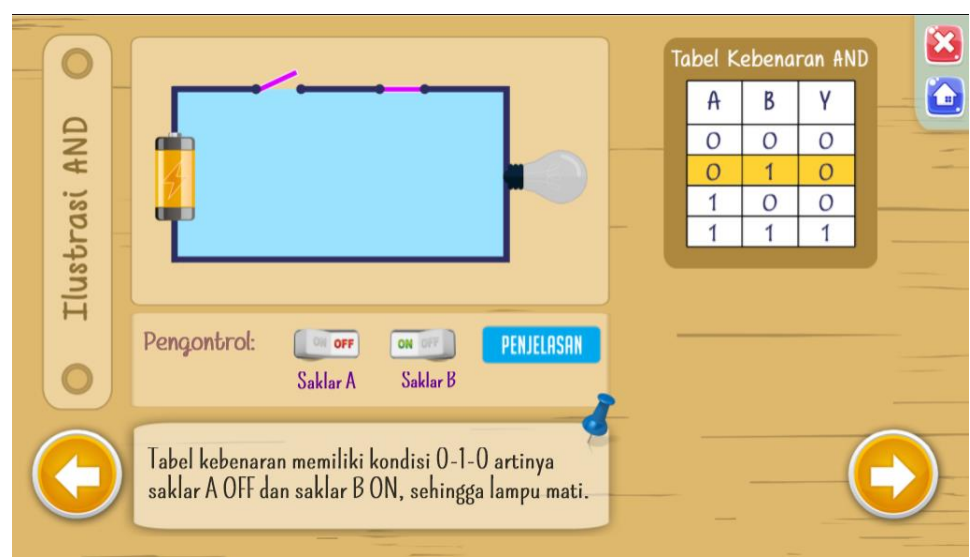

Fig. 2. Simulation interface of AND Logic Gate. 


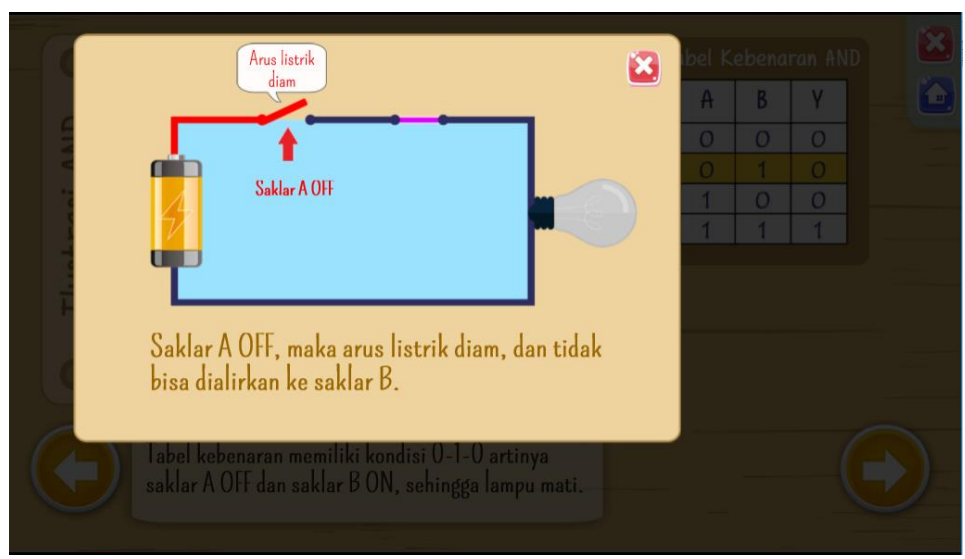

Fig. 3. Pop-up explanation of the AND Logic Gate simulation interface.

\subsection{Comparative Analysis of Gain and Student Assessment of CAI}

Based on the pretest, the use of CAI, and the results of the posttest, a comparative analysis was carried out between the results of the gains obtained by students and the responses/assessments of the media by students. The study is intended to determine the effect of simulation type CAI on student learning outcomes. Table 1 presents information related to improved learning outcomes in learning using simulation media and student responses to the developed simulation media.

Table 1. Cognitive and Media Assessment Description.

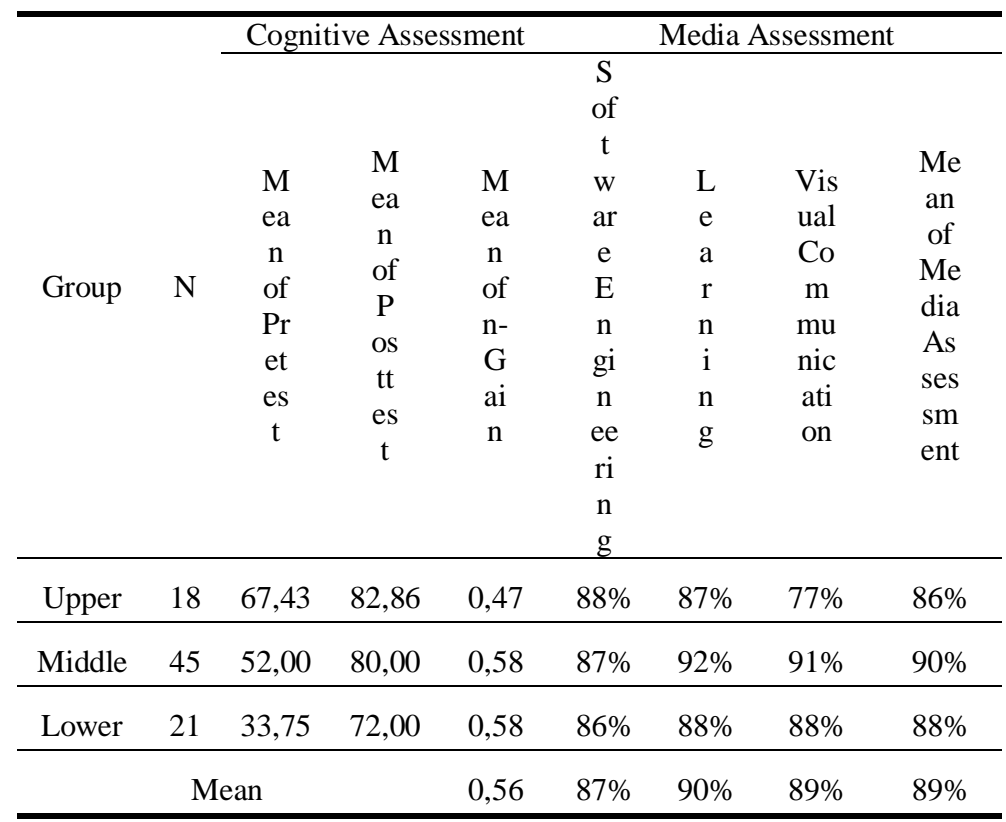


To find out more about the role of simulation media in improving student learning outcomes, interviews were carried out to three students who represented the relationship between gain values and mean responses of students to simulation media. The first student (respondent 20) selected is a student who enters the middle group students, gets a gain of 0.71 (included in the high category), and gives an excellent media response on almost every item. The second student (respondent 19) selected as the student who entered the upper group students, got a gain of 0.58 (included in the medium category) and gave a relatively high response on each of the media items. And the last student is respondent 9, which included in the lower group students, get a gain of 0.25 (including the low category), and gives a pretty good response in the evaluation of media simulation.

Table 2 presents some situations of the relationship between the results of the gain and student assessment of multimedia. Interviews conducted with Respondents expressed the opinion that the media used were very good because the simulations provided were sufficient to provide initial information regarding operations occurring within the logic gate that were sometimes only understood abstractly. Respondent 20 also stated that the simulation media is exciting, simple, easy to understand, and can respond to everything instructed by the user.

Table 2. Gain results and media assessments.

\begin{tabular}{ccccccc}
\hline \multirow{2}{*}{ Identity } & \multicolumn{3}{c}{ Cognitive assessment } & \multicolumn{2}{c}{ Media assessment } \\
\cline { 2 - 6 } & Pretest & Posttest & n-gain & $\begin{array}{c}\text { Software } \\
\text { Engineering }\end{array}$ & Learning & $\begin{array}{c}\text { Visual } \\
\text { communication }\end{array}$ \\
\hline Resp. 20 & 44 & 84 & 0,71 & $90 \%$ & $97 \%$ & $97 \%$ \\
\hline Resp. 19 & 52 & 80 & 0,58 & $83 \%$ & $90 \%$ & $83 \%$ \\
\hline Resp. 9 & 68 & 76 & 0,25 & $73 \%$ & $77 \%$ & $74 \%$ \\
\hline
\end{tabular}

Meanwhile, Respondent 19 stated that the simulation is easy to understand because animated explanations of each condition accompany it, but the seven logic gates simulated with the same type, which makes the user bored. The lack of challenge scenarios in the simulation media also caused Respondents 19 to be less enthusiastic about the developed media. In contrast to Respondents 20 and Respondents 19, Respondent 9 stated that the simulation was still challenging to understand because there was an error in the text displayed, some offerings still needed confirmation from the teacher.

\section{Discussion}

The gain results are not in the high category because they caused by several factors including limited time and opportunities for students to interact with the media developed. Because it deals with new technology, ideally, the intensity of student meetings with developed technology can be more multiplied so that students are familiar with the plot and features of the media developed. Another factor that occurs in the media is that several computers do not support the type of font loaded by researchers on the developed CAI, so the font type changes to the default font of the computer with a very large size and covers the image. Also, some students stated the lack of variation in the illustration of the Logic Gate material, so students were bored using the media because they had to do simulations repeatedly. 
Meanwhile, the gain is not in the low category because some excess media cause it. These advantages obtained from the opinions of students included in the instruments of student assessment of CAI, including:

1. CAI can make students better understand the concept of Logic Gates material that is abstract because simulations visualize the content on electric circuit illustrations that made as if users are in a real situation.

2. Learning becomes more interesting and not monotonous because the presentation of the material is accompanied by animations that can make it easier for students to remember the content.

3. CAI can make students more active when learning because learning is done in two directions, where CAI can deliver material to students, and students can conduct simulations on CAI.

From the results of student assessments, the simulation type CAI is stated to be very good for use in Computer Systems subjects, but it hoped that there would-be researchers in the next generation who can redevelop this simulation type CAI by adding transistors instead of switches [6], because there is difference in simplicity of the circuit. Transistors used as amplifiers, circuit breakers and connectors, and voltage stabilization. The type of transistor to implement a simple switch that often used is MOSFET (Metal Oxide Semiconductor Field Effect Transistor). There are two types of MOSFET transistors, namely negative channel MOS transistor (nMOS) and positive channel MOS transistor (pMOS). In the nMOS transistor, when the gate gave a forward voltage (high), the transistor will be active. In pMOS, the opposite applies, i.e., when the gate legs gave a forward voltage (high), then the transistor is not active.

By using a transistor instead of a switch, the gate simulation contained in the CAI can be implemented in a real situation, which is making the actual electrical circuit.

\section{References}

[1] L. Traynor, "Effects of Computer-Assisted-Instruction on Different Learners," Journal of Instructional Psychology, vol. 30, no. 2, pp. 137-143, 2003.

[2] I. Joy, U. U. Christian, O. D. Omenka, E. E. Dorathy, E. A. Benjamin, S. O. Egboja, E. Bernard and I. P. Aondona, "Comparative Study of Computer Assisted Instruction (CAI) and Conventional Strategyin Enhancing Students' Academic Performance in Senior Secondary schools in Oju Local Government Area," International Journal of Science and Research Methodology, vol. 7, no. 1, pp. 138-148, 2017

[3] Heinich, M. Molenda and J. D. Russell, Instructional Media and the New Technologies of Instruction, 4th ed., New York: Macmillan Coll Div, 1993.

[4] Robinson, Simulation: The Practice of Model Development and Use, Chichester, West Sussex England: John Wiley \& Sons Ltd, 2004.

[5]. Crellin, M. Adda, and E. Duke-Williams, "The Use of Simulation in Digital Forensics Teaching," in 11th Annual Conference of the Higher Education Academy, Information and Computer Science Group, Durham, 2010.

[6] Brown and Z. Vranesic, Fundamentals of digital logic with VHDL Design, New York: McGrawHill, 2009. 